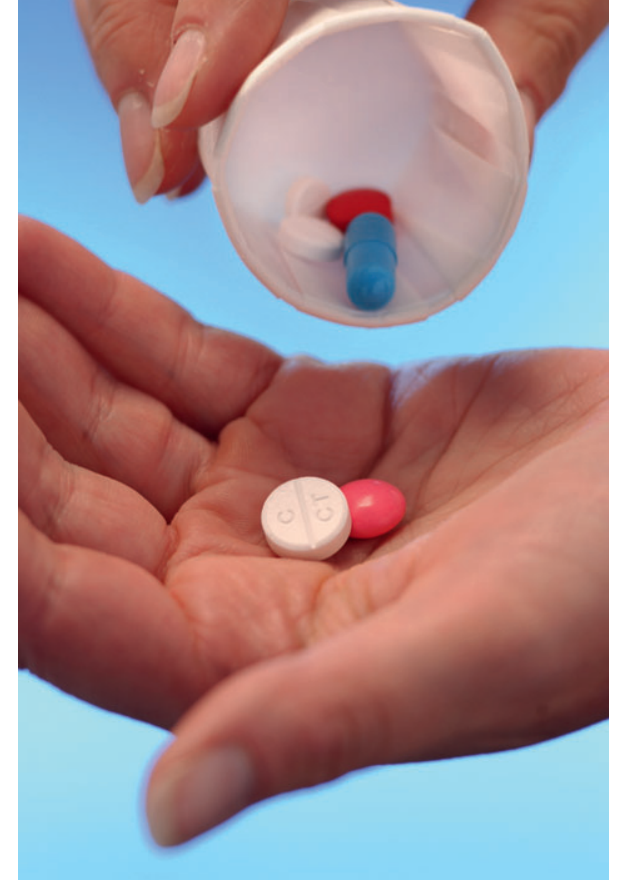

\title{
Experiential learning about medication adherence
}

\section{Jennifer N Stojann ${ }^{1}$, Margaret Wolff ${ }^{2}$, Stacie Buckler ${ }^{3}$, Jason Kahnn ${ }^{1}$, Sally A Santen ${ }^{2}$ and Michelle Daniel ${ }^{2}$}

${ }^{1}$ Internal Medicine and Pediatrics, University of Michigan, Ann Arbor, Michigan, USA ${ }^{2}$ Emergency Medicine, University of Michigan, Ann Arbor, Michigan, USA ${ }^{3}$ Office of Medical Student Education, University of Michigan, Ann Arbor, Michigan, USA

$\mathrm{M}$ edication non-adherence contributes to poor health outcomes and causes financial burden. ${ }^{1}$ With the recognition of the difficulty of medication adherence, there has been a shift in educational practices. The judgemental nomenclature of 'noncompliance' is gradually being replaced by 'non-adherence', and greater emphasis is being placed on the importance of shared decision making.

Why is there a need for change? The term noncompliance implies patients should simply follow their doctor's orders, rather than actively engage as participants in their own health care. The term can be stigmatising, derogatory and negatively affect the relationships that patients have with their current and future health care providers. $^{2}$ Despite this, many practising doctors persist in using the term noncompliant, fail to inquire about barriers to adherence, believe it is the patient's responsibility to follow their orders and become frustrated when patients are non-adherent. ${ }^{3}$

To appropriately counsel patients and forge a therapeutic alliance, doctors-in-training must come to appreciate barriers to adherence rather than place blame, recognising that adherence is influenced by individual, social and environmental factors. ${ }^{4}$ Training about adherence should ideally begin early in medical school.

Using an experiential approach, we designed a curriculum to help preclinical medical students understand the difficulties of adherence and the challenges patients face through a mock prescription programme. The design was informed by the Kolb learning cycle in which educational activities move the learner from a definition of personal meaning to the
The term noncompliance implies patients should simply follow their doctor's orders 


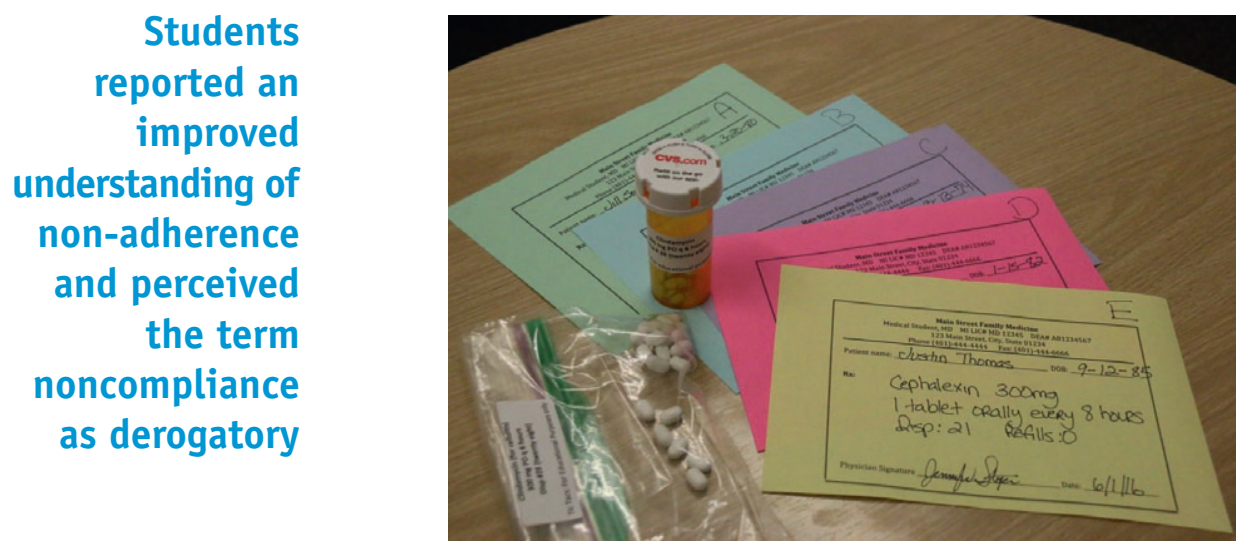

Figure 1. Five mock prescriptions, varied by type and number of times per day

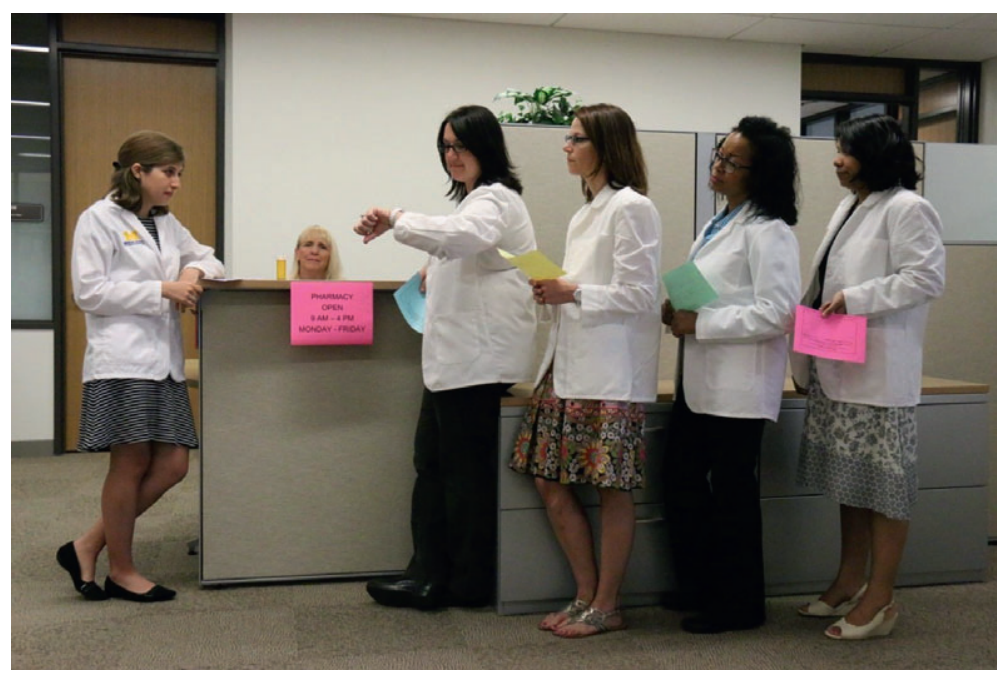

Figure 2. Queue for the mock pharmacy

acquisition of new knowledge, and finally to the practical application, synthesis and extension of this knowledge. ${ }^{5}$

First-year medical students, enrolled in a required clinical skills course focused on historytaking, communication and physical examination skills, as well as sociobehavioural topics, participated in an exercise demonstrating the potential barriers that patients encounter when taking a single prescription medicine. Each student received one of five mock prescriptions consisting of Tic-Tac ${ }^{\text {t" }}$ candy (Figure 1). The prescription type and number of times taken per day varied, creating different experiences to discuss during a debriefing session.

Students took their prescription to the mock pharmacy, located in the medical school education building, to be filled course administrator). The pharmacy was only open during business hours, thereby creating a barrier to obtaining the prescription, with students needing to find time in their busy schedules to fill it.

Students had varied pharmacy experiences. Some students found long queues (Figure 2), forcing them to return later. Others were told that the expense of the medication exceeded what they could afford. These students were forced to e-mail the mock doctor (course director) for a less expensive prescription and then return to the pharmacy to have it filled.

The following week, students participated in a small group by the mock pharmacist (the discussion about medication non-adherence, facilitated by course faculty members. Students reflected upon their personal experience of taking the 'medication', and any barriers they encountered. They brainstormed ways that doctors can engage patients in shared decision making and collaborate to remove barriers. Discussion surrounded strategies that help patients to remember to take medications, including recommending the use of a pillbox, and prescribing medications with less frequent dosing, the importance of providing both written and verbal medication instructions, and verifying patient understanding, and removing barriers to getting prescriptions filled, such as confirming patients can afford medications and obtaining transportation to the pharmacy.

After the exercise, students reported an improved understanding of non-adherence and perceived the term noncompliance as derogatory. Students were less likely to find it acceptable to label patients as noncompliant. They no longer believed that a patient should be able to follow medication instructions, remember to take medications as prescribed and remember to take medications multiple times a day simply because that was what the doctor ordered. After participation, students' understanding of the difficulty of getting prescriptions filled increased, as did their understanding of the challenges faced in taking medications as prescribed.

This innovation suggests that participating in a simple experiential learning exercise over a short period of time can improve preclinical medical students' attitudes regarding non-adherence and their recognition of the myriad of barriers that patients encounter. Merely hearing about non-adherence may lead students to believe that taking medications as directed is a simple process 
unless they experience it for themselves. Engaging in both active learning and reflection has the potential to leave a more memorable impression.

Although we believe this exercise is valuable to lay a solid foundation about non-adherence in preclinical learners, experiencing barriers to adherence may be even more impactful during clinical training. In the future, we plan to implement a similar prescription exercise for more advanced learners. They will be asked to take a comparable medication regimen as one of their medical or surgical patients, which often includes taking more than 10 different medications at specific times of the day. Learners will be asked to reflect on additional barriers to adherence created by such polypharmacy, and gain an even greater appreciation of the patient experience.

\section{REFERENCES}

1. Vermeire $E$, Hearnshaw $H$, Van Royen P, Denekens J. Patient adherence to treatment: three decades of research. A comprehensive review. J Clin Pharm Ther 2001;26:331-342.
2. Osterberg L, Blaschke T. Adherence to medication. N Engl J Med 2005;353:487-497.

3. Tarn DM, Mattimore TJ, Bell DS, Kravitz RL, Wenger NS. Provider views about responsibility for medication adherence and content of physician-older patient discussions. J Am Geriatr Soc 2012;60:1019-1026.

4. Donovan JL, Blake DR. Patient noncompliance: deviance or reasoned decision-making? Soc Sci Med 1992;34:507-513.

5. Armstrong E, Parsa-Parsi R. How can physicians' learning styles drive educational planning? Acad Med 2005;80:680-684.

Corresponding author's contact details: Jennifer N Stojan, Internal Medicine and Pediatrics, University of Michigan, 1041 Maiden Lane, Ann Arbor, Michigan 48105, USA. E-mail: jstojan@umich.edu

Funding: None.

Conflict of interest: None.

Acknowledgements: The authors would like to acknowledge Dr Paula Ross for all of her help on this manuscript.

Ethical approval: This study received IRB exempt from review status from the University of Michigan IRB.

doi: $10.1111 /$ tct.12645 